\title{
GLAD!
}

Revue sur le langage, le genre, les sexualités

$02 \mid 2017$

Varia

\section{Les matérialités discursives du sexe. La construction et la déstabilisation des évidences du genre dans les discours sur les sexes atypiques}

Résumé de thèse

The Discursive Materialities of Sex. Making and Undermining Gender Evidence

Through Discourses on Atypical Sexes

\section{Noémie Marignier}

\section{OpenEdition}

Journals

Édition électronique

URL : http://journals.openedition.org/glad/503

DOI : $10.4000 /$ glad.503

ISSN : 2551-0819

Éditeur

Association GSL

Référence électronique

Noémie Marignier, «Les matérialités discursives du sexe. La construction et la déstabilisation des

évidences du genre dans les discours sur les sexes atypiques », GLAD! [En ligne], 02 | 2017, mis en ligne le 01 juin 2017, consulté le 21 janvier 2021. URL : http://journals.openedition.org/glad/503 ; DOI : https://doi.org/10.4000/glad.503

Ce document a été généré automatiquement le 21 janvier 2021.

La revue GLAD! est mise à disposition selon les termes de la Licence Creative Commons Attribution Pas d'Utilisation Commerciale - Pas de Modification 4.0 International. 


\section{Les matérialités discursives du sexe. $\mathrm{La}$ construction et la déstabilisation des évidences du genre dans les discours sur les sexes atypiques}

Résumé de thèse

The Discursive Materialities of Sex. Making and Undermining Gender Evidence

Through Discourses on Atypical Sexes

Noémie Marignier

\section{RÉFÉRENCE}

N. Marignier. 2016. Les matérialités discursives du sexe. La construction et la déstabilisation des évidences du genre dans les discours sur les sexes atypiques. Thèse de doctorat en Sciences du Langage, sous la direction de Luca Greco et Marie-Anne Paveau, Université Paris 13 Sorbonne Paris Cité. [en ligne : tel.archives-ouvertes.fr/tel-01418262]

Ma thèse porte sur les discours relatifs aux variations du développement du sexe. Elle trouve son point de départ dans les recherches féministes et queer qui ont montré que non seulement le genre, mais aussi le sexe sont des constructions discursives (Butler 1993) : dans cette perspective, les sexes mâle et femelle ne sont pas un donné biologique ou naturel (Kraus 2000), mais sont produits et reproduits dans les pratiques langagières selon un principe de binarité. L'existence de personnes intersexes remet alors en cause cette organisation binaire des sexes (Fausto-Sterling 2012). Il s'agit donc de s'intéresser aux discours sur les sexes atypiques et de les considérer comme un lieu où la différence binaire des sexes est possiblement déstabilisée ou au contraire produite et réaffirmée par les discours. 
2 Cette thèse s'inscrit dans le domaine de l'analyse du discours avec un double ancrage théorique. D'une part, je m'inscris dans la continuité de l'analyse de discours dite française, en faisant dialoguer la théorie du discours avec les études de genre. Il s'agit de travailler certains concepts clés de l'analyse du discours - tels que ceux de formation discursive, de préconstruit ou d'idéologie (Pêcheux 1975, Henry 1977) - au prisme des études de genre afin de fournir une assise théorique solide à l'analyse des discours du sexe. Ce retravail permet alors une réflexion sur la manière dont se constituent les évidences concernant le sexe et dont se réalise l'interpellation des sujets sexués et genrés à l'interface de la langue et des idéologies de genre. D'autre part, ma thèse adopte la perspective des Gender \& Language Studies anglophones dont les recherches portent sur les questions de pratiques de catégorisations et de construction des identités de genre (Eckert \& McConnell-Ginet 1992, Livia \& Hall 1997, Bucholtz \& Hall 2005) aussi bien que sur la part langagière du désir (Cameron \& Kulick 2003). Il s'agit alors de faire dialoguer ce champ d'études avec les approches françaises du discours afin de contribuer à développer, dans le champ francophone, des recherches linguistiques sur le genre et les sexualités mettant au centre les questions de constitution du sens et de matérialités langagières.

3 Trois corpus ont été constitués afin de mener des analyses sur la construction discursive des sexes. D'une part, j'ai recueilli un corpus de discours médicaux constitué d'articles scientifiques parus entre 2000 et 2014 et de dossiers médicaux d'enfants au sexe atypique nées entre 1995 et 2013. Une autre partie du corpus est composée de discours militants : il s'agit d'un corpus de discours numériques recueillis sur les sites web des différentes associations de personnes concernées par les variations du développement du sexe ainsi que sur les forums qui leur sont associés. Enfin, j'ai constitué un corpus de discours pornographiques, où des désirs pour les sexes atypiques étaient exprimés, à partir de sites web consacrés aux sexualités alternatives ou de tubes pornographiques mainstream.

4 J'ai mené des analyses qualitatives des observables prélevés dans le corpus. Ces analyses portent sur la manière dont les différentes locuteur.ices mobilisent les ressources sémantiques, lexicales, énonciatives et pragmatiques de la langue dans leurs pratiques discursives afin de produire les sens du sexe, de créer les identités sexuées, mais aussi de stabiliser ou déstabiliser les idéologies de genre dans des mouvements constants de naturalisation (figements, effets d'évidence, stéréotypies) et de dénaturalisation (non-coïncidences des dires, créations lexico-syntaxiques) de la différence des sexes. Finalement, ma thèse montre que les sens du sexe sont toujours en train de se faire et de se défaire dans les discours: produits par l'idéologie hétéronormative, affirmés ou subvertis par les constitutions subjectives, reconfigurés par les discours du désir. 


\section{BIBLIOGRAPHIE}

BUCHOLTZ, M., \& HALL, K. (2005). «Identity and interaction : A sociocultural linguistic approach » Discourse studies, 7(4-5), 585-614.

BUTLER, J. (1993). Bodies that matter : on the discursive limits of «sex ». New York : Routledge.

CAMERON, D., \& KULICK, D. (2003). Language and sexuality. Cambridge : Cambridge University Press.

ECKERT, P., \& McCONNELL-GINET, S. (1992). « Think practically and look locally : Language and gender as community-based practice » Annual review of anthropology, 21, 461-490.

FAUSTO-STERLING, A. (2012). Corps en tous genres : la dualité des sexes à l'épreuve de la science. (E. Peyre, C. Vidal, \& J. Wiels, Éd., O. Bonis \& F. Bouillot, Trad.) Paris : Éditions la Découverte.

HENRY, P. (1977). Le mauvais outil : langue, sujet et discours. Paris : Klincksieck.

KRAUS, C. (2000). «La bicatégorisation par sexe à l'“épreuve de la science" : le cas des recherches en biologie sur la détermination du sexe chez les humains ", in GARDEY Delphine \& LÖWY Ilana (éd.), L'invention du naturel : les sciences et la fabrication du féminin et du masculin. Paris : éd. des archives contemporaines.

LIVIA, A., \& HALL, K. (1997). Queerly phrased : Language, gender, and sexuality. New York : Oxford University Press.

PÊCHEUX, M. (1975). Les vérités de La Palice : linguistique, sémantique, philosophie. Paris : F. Maspero.

\section{INDEX}

Thèmes : Actualités

Keywords : sexes, discourse practices, ideologies, discursive formations, identities

Mots-clés : sexes, pratiques discursives, idéologie, formations discursives, identités 